\title{
Estudo morfológico das espécies Eimeria alijevi, E. arloingi e E. ninakohlyakimovae de caprinos leiteiros jovens de um criatório na microrregião Lagos, $\mathbf{R J}$
}

\author{
Morphologycal study of Eimeria alijevi, E. arloingi and E. \\ ninakohlyakimovae of young goats from a dairy farm in microrregion \\ Lagos, of Rio de Janeiro
}

\author{
Izabella Cabral Hassum, * Rita de Cássia Alves Alcantara de Menezes**
}

\begin{abstract}
Resumo
Foram examinadas amostras de fezes de caprinos jovens, machos e fêmeas, integrantes de um rebanho leiteiro, oriundo do município de Saquarema, a fim de pesquisar a presença das espécies Eimeria alijevi, E. arloingi e E. ninakohlyakimovae. Oocistos esporulados, de cada espécie, foram identificados com base na morfologia, índice morfométrico (IM) e características das estruturas internas. Os oocistos esporulados das Eimeria alijevi, E. arloingi e E. ninakohlyakimovae apresentaram, respectivamente, as seguintes medidas: $19,93 \pm 1,70$ por $18,38 \pm 1,79 \mathrm{~mm}$ com IM 1,09 $\pm 0,06$; $28,84 \pm 2,20$ por $20,30 \pm 1,35 \mathrm{~mm}$

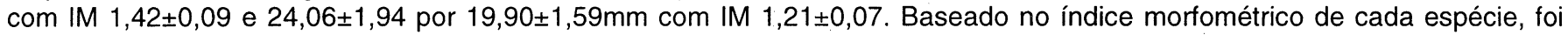
possível determinar a forma dos oocistos esporulados: subesférica para $E$. alijevi, elipsoidal para $E$. arloingi e subesférica a elipsoidal para $E$. ninakohlyakimovae.
\end{abstract}

Palavras-chave: Eimeria alijevi; Eimeria arloingi e Eimeria ninakohlyakimovae; caprinos leiteiros; morfologia.

\begin{abstract}
Were examined fecal samples of young goats, male and female from a dairy farm in Saquarema county, microrregion Lagos, in Rio de Janeiro State to research Eimeria alijevi, E. arloingi and E. ninakohlyakimovae species presence. Sporulated oocysts of each species were identified based on the morphology, shape index (SI) and internal features. The sporulated oocysts of Eimeria alijevi, E. arloingi and E. ninakohlyakimovae respectively presented the following measures: $19,93 \pm 1,70$ by $18,38 \pm 1,79 \mathrm{~mm}$ with SI $1,09 \pm 0,06 ; 28,84 \pm 2,20$ by $20,30 \pm 1,35 \mathrm{~mm}$ with SI $1,42 \pm 0,09$ and $24,06 \pm 1,94$ by $19,90 \pm 1,59 \mathrm{~mm}$ with $\mathrm{SI}$ $1,21 \pm 0,07$. Based on the shape index of each species, it was possible to determinate the oocysts shape: subspherical for $E$. alijevi, ellipsoidal for $E$. arloingi and subspherical to ellipsoidal for $E$. ninakohlyakimovae.
\end{abstract}

Keywords: Eimeria alijevi; Eimeria arloingi; Eimeria ninakohlyakimovae; dairy goats; morphology.

\section{Introdução}

A eimeriose é uma doença que afeta, principalmente, animais jovens, causando muitas vezes perdas econômicas em decorrência da alta mortalidade nestes animais, além da morbidade que se reflete no retardamento do crescimento.

Lloyd e Soulsby (1978) constataram que em 100\% das amostras de fezes de caprinos examinados estavam presentes oocistos do gênero Eimeria, sendo que as maiores percentagens foram para animais com menos de seis meses de idade. A patogenicidade determinada por este protozoário é variável de acordo com a espécie que esteja parasitando o animal, já que a contaminação é multiespecífica (Santana e Pereira, 1984, Bomfim e Lopes, 1994). Desta forma, a identificação das espécies de Eimeria é de suma importância.
A morfologia dos oocistos de ruminantes pode ser utilizada para diferenciar as espécies e ainda servir como base para estudos epidemiológicos (Joyner e Long, 1974, Long e Joyner, 1984).

O objetivo deste estudo foi contribuir com parâmetros morfológicos para diagnóstico das espécies de Eimeria parasitas dos caprinos jovens.

\section{Material e métodos}

Foram utilizados caprinos leiteiros jovens de até 180 dias de idade, machos e fêmeas, pertencentes a um criatório particular situado no município de Saquarema, na microrregião Lagos, Estado do Rio de Janeiro. Os animais, em sua maioria sem raça definida, eram mantidos junto aos animais adultos em sistema de manejo semi-intensivo.

* Curso de Pós-Graduação em Medicina Veterinária-Parasitologia Veterinária, Universidade Federal Rural do Rio de Janeiro, Seropédica, RJ, Brasil, 23890-000.

** Departamento de Parasitologia Animal, Instituto de Biologia, UFRRJ 
As amostras foram obtidas em três coletas fecais, diretamente da ampola retal dos animais, durante o período seco (maio, junho e agosto de 1996).

As fezes foram processadas pela técnica de centrífugoflutuação, de acordo com Menezes e Lopes (1995), para conferir a presença de oocistos de Eimeria e, em seguida, colocadas para esporular em bicromato de potássio a 2,5\%. Após a esporulação, a identificação de cada espécie de Eimeria foi baseada na forma, medidas e estruturas internas dos oocistos, observados por meio de microscópio óptico e ocular micrométrica, tendo-se como referência os trabalhos de Levine (1985) e Norton (1986).

Os exames laboratoriais foram realizados na Estação para Pesquisas Parasitológicas W. O. Neitz, do Departamento de Parasitologia Animal da Universidade Federal Rural do Rio de Janeiro.

\section{Resultados e discussão}

Na Figura 1 encontram-se os oocistos esporulados das espécies estudadas e na Tabela 1 estão apresentadas as características morfológicas dos oocistos e esporocistos de $E$. alijevi, E. arloingi e E. ninakohlyakimovae.

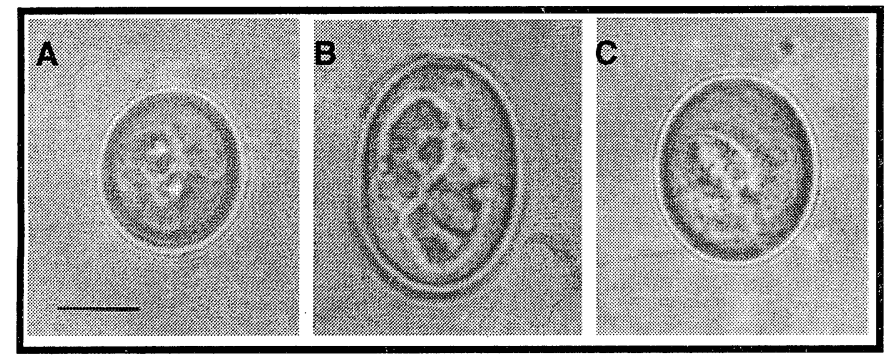

Figura 1: Oocistos esporulados de Eimeria alijevi (A), E. arloingi (B) e $E$. ninakohlyakimovae (C) em solução saturada de açúcar $(-=10 \mu \mathrm{m})$.
Menezes e Lopes (1997) encontraram oocistos de E. alijevi de menor tamanho, porém com índice morfométrico muito próximo. Já Singh (1962) observou oocistos de $E$. alijevi de menor tamanho que os encontrados no presente estudo, além de um índice morfométrico também menor. Em contrapartida, para E. ninakohlyakimovae este mesmo autor verificou oocistos de tamanhos semelhantes, com IM menor. Teixeira Filho (1998), estudando a E. ninakohlyakimovae em caprinos de uma propriedade na microrregião Fluminense do Grande Rio, observou oocistos de tamanho semelhante aos encontrados no presente trabalho. No entanto, quando esse autor comparou os oocistos provenientes de animais jovens e adultos, verificou variação intraespecífica, com um certo grau de pleomorfismo.

No ano de 1963, em Madhya Pradesh, Shah e Joshi revelaram oocistos das três espécies de Eimeria com tamanhos semelhantes aos citados neste estudo, diferindo apenas no tamanho da parede dos oocistos de E. alijevi, que para os autores foi menor.

Lima (1980) encontrou oocistos de $E$. alijevi menores, porém apresentando o mesmo índice morfométrico, enquanto que para as outras duas espécies seus resultados coincidiram com os do presente trabalho.

No Estado de Pernambuco, Santana e Pereira (1984) obtiveram oocistos menores de $E$. alijevi, porém os oocistos de $E$. ninakohlyakimovae tiveram medidas semelhantes aos relacionados neste estudo.

As medidas dos oocistos de E. arloingi e E. ninakohlyakimovae foram próximas às apresentadas por Alvarado et al. (1990), sendo que para $E$. alijevi, as medidas foram menores.

Em 1994, Bomfim e Lopes observaram oocistos das três espécies de Eimeria menores que os aqui descritos, onde apenas a espessura da parede dos oocistos de $E$. arloingi foi semelhante.

Tabela 1: Características morfológicas e medidas dos oocistos e esporocistos das espécies Eimeria alijevi, E. arloingi e E. ninakohlyakimovae de caprinos leiteiros jovens de uma criação localizada na microrregião Lagos, RJ.

\begin{tabular}{|c|c|c|c|c|c|c|c|}
\hline \multicolumn{5}{|c|}{ Oocisto } & \multicolumn{3}{|c|}{ Esporocisto } \\
\hline Espécies & $\begin{array}{c}\text { Tamanho }(\mu \mathrm{m})^{\mathrm{b}} \\
(\mathrm{DM} / \mathrm{dm})^{\mathrm{c}}\end{array}$ & $\begin{array}{c}\text { Índice } \\
\text { morfométrico }\end{array}$ & Micrópila & Calota $(\mu \mathrm{m})$ & $\begin{array}{c}\text { Tamanho }(\mu \mathrm{m}) \\
(\mathrm{DM} / \mathrm{dm})\end{array}$ & Forma & $\begin{array}{l}\text { Corpo de } \\
\text { Stieda }\end{array}$ \\
\hline E. alijevi $(51)^{a}$ & $\begin{array}{l}9,93 \pm 1,70 / \\
18,38 \pm 1,79\end{array}$ & $1,09 \pm 0,06$ & $\begin{array}{l}\text { Vestigial } \\
\text { ausente }\end{array}$ & Ausente & $\begin{array}{l}9,36 \pm 1,41 / \\
5,90 \pm 0,80\end{array}$ & Subesférica & Ausente \\
\hline E. arloingi (64) & $\begin{array}{l}28,84 \pm 2,20 / \\
20,30 \pm 1,35\end{array}$ & $1,42 \pm 0,09$ & Presente & $5,10 \pm 0,97$ & $\begin{array}{c}11,58 \pm 1,75 / \\
6,66 \pm 0,82\end{array}$ & Alongada & $\begin{array}{l}\text { Vestigial } \\
\text { ausente }\end{array}$ \\
\hline $\begin{array}{c}\text { E. ninakohlyakimovae } \\
\qquad(68)\end{array}$ & $\begin{array}{r}24,06 \pm 1,94 / \\
19,90 \pm 1,59\end{array}$ & $1,21 \pm 0,07$ & Presente & Ausente & $\begin{array}{c}10,62 \pm 1,49 / \\
6,49 \pm 0,83\end{array}$ & Oval & Presente \\
\hline
\end{tabular}

a Número de oocistos medidos entre parênteses, 'bedidas em $x \pm$ Dsd, 'Diâmetro maior (DM) e diâmetro menor (dm).

Neste estudo, considerou-se a soma das duas camadas, como a espessura da parede dos oocistos e verificaram-se as seguintes medidas: E. alijevi $1,35 \pm 0,34 \mu \mathrm{m}, E$. arloingi $1,48 \pm 0,25 \mu \mathrm{m}$ e. E. ninakohlyakimovae $1,32 \pm 0,31 \mu \mathrm{m}$. Dentre as três espécies, apenas $E$. arloingi possui capuz polar, o qual mediu $5,10 \pm 0,97 \mu \mathrm{m}$.
Menezes e Lopes (1995) encontraram para E. arloingi resultados próximos aos do presente estudo, onde o capuz polar foi igual a $5,23 \pm 0,83 \mu \mathrm{m}$.

Baseado no índice morfométrico, foi possível concluir que o formato dos oocistos de E. alijevi foi subesférico, E. arloingi elipsoidal e E. ninakohlyakimovae de subesférico a elipsoidal. 


\section{Referências bibliográficas}

ALVARADO, R., PITTY, B., MORALES, S. Géneros de helmintos gastrointestinales y especies de coccidios de ganado caprino de Costa Rica. Cienc. Vet. (Heredia), n. 12, p. 25-27, 1990.

BOMFIM, T. C. B. do \& LOPES, C. W. G. Levantamento de parasitos gastrintestinais em caprinos da região serrana do estado do Rio de Janeiro. Rev. Bras. Parasitol. Vet., n. 3, p. 119-124, 1994.

JOYNER, L. P., LONG, P. L. The specific characters of the Eimeria, with special reference to the coccidia of the fowl. Avian Pathol., n. 3, p. 145-157, 1974.

LEVINE, N. D. Veterinary Protozoology. Ames : lowa State University Press, 414 p, 1985.

LIMA, J. D. Prevalence of coccidia in domestic goats from Illinois, Indiana, Missouri and Wisconsin. Int. Goat and Sheep Res., n. 3, p. 234-241, 1980.

LLOYD, S., SOULSBY, E. J. L. Survey of parasites in dairy goats. Am. J. Vet. Res., v. 39, p. 1057-1059, 1978.

LONG, P. L., JOYNER, L. P. Problems in the identification of species of Eimeria. J. Protozool., v. 31, p. 535-541, 1984.

MENEZES, R. de C. A. A. de, LOPES, C. W. G. Epizootiologia da Eimeria arloingi em caprinos na microrregião serrana fluminense, Rio de
Janeiro, Brasil. Rev. Univ. Rural, Sér. Ciênc. da Vida, v. 17, p. 5-12, 1995.

MENEZES, R. de C. A. A. de, LOPES, C.W. G. E. alijevi(APICOMPLEXA: EIMERIIDAE) em caprinos leiteiros na microrregião Serrana Fluminense, RJ. Rev. Bras.Parasitol. Vet., n. 6, p. 69-73, 1997.

NORTON, C. C. Coccidia of the domestic goat Capra hircus, with notes on Eimeria ovinoidalis and E. bakuensis (syn. E. ovina) from the sheep Ovis aries. Parasitology, v. 92, p. 279-289, 1986.

SANTANA, A. F. de, PEREIRA, I. H. de O. Espécies de Eimeriaparasitas de caprinos no sertão de Pernambuco. Cad. Ômega Univ. Fed. Rural P.E. Sér. Vet., Recife,n. 1, p. 15-23, 1984.

SHAH, H. L., JOSHI, S. C. Coccidia (PROTOZOA: EIMERIIDAE) of goats in Madhya Pradesh, with descriptions of the sporulated oocysts of eight species. J. Vet. \& Anim. Hus. Res., n. 7, p. 920, 1963.

SINGH, P. P. On eimerian oocyst in indian goat; na exogenous study. Agra University Journal of Research, v. 13, p. 233-238, 1962.

TEIXEIRA FILHO,W. L. 1998. Aspectos morfobiológicos da Eimeria ninakohlyakimovae Yakimoff \& Rastegaieff, 1930 (Apicomplexa: Eimeriidae) procedentes de caprinos da microrregião Fluminense do Grande Rio, Estado do Rio de Janeiro. 46 p. Tese (Mestrado) Universidade Federal Rural do Rio de Janeiro. 